\title{
Response of microbial community structure and metabolic profile to shifts of inlet VOCs in a gas-phase biofilter
}

\author{
Lichao Lu', Guangchun Wang ${ }^{3}$, Marvin Yeung ${ }^{1}$, Jinying $\mathrm{Xi}^{1}{ }^{12^{*}}$ and Hong-Ying Hu${ }^{1,2}$
}

\begin{abstract}
The effects of inlet VOCs (Volatile Organic Compounds) shifts on microbial community structure in a biofiltration system were investigated. A lab-scale biofilter was set up to treat eight VOCs sequentially. Short declines in removal efficiency appeared after VOCs shifts and then later recovered. The number of OTUs in the biofilter declined from 690 to 312 over time. At the phylum level, Actinobacteria and Proteobacteria remained dominant throughout the operation for all VOCs, with their combined abundance ranging from 60 to 90\%. The abundances of Planctomycetes and Thermi increased significantly to $20 \%$ and $5 \%$, respectively, with the intake of non-aromatic hydrocarbons. At the genus level, Rhodococcus was present in the highest abundance $(\geq 10 \%)$ throughout the experiment, indicating its wide degradability. Some potential degraders were also found; namely, Thauera and Pseudomonas, which increased in abundance to $19 \%$ and $12 \%$ during treatment with ethyl acetate and toluene, respectively. Moreover, the microbial metabolic activity declined gradually with time, and the metabolic profile of the toluene-treating community differed significantly from those of other communities.
\end{abstract}

Keywords: VOCs, Biofiltration, Microbial community, Metabolic profile

\section{Introduction}

There has been growing interest in control of the emission of volatile organic compounds (VOCs) because of their potential to harm the environment and human health toxicity (Zheng et al. 2013). Recently, waste gases emitted in high volume containing complex VOCs at low concentration have hindered performance enhancement of related treatments (Cheng et al. 2016). This has led to increased interest in biofiltration systems, which have advantages of high efficiency, minimal secondary pollution and low costs (Khan and Ghoshal 2000).

Microorganisms play an important role in biodegradation, having direct interactions with contaminants. In previous studies, significant shifts in the bacterial community were observed during biodegradation, especially in the initial period (Qiu et al. 2013). Increasing doses

\footnotetext{
*Correspondence: xijinying@tsinghua.edu.cn

${ }^{1}$ Environmental Simulation and Pollution Control State Key Joint Laboratory, School of Environment, Tsinghua University, Beijing, China Full list of author information is available at the end of the article
}

also impact microbial communities ( $\mathrm{Li}$ et al. 2017). Efforts were also paid on microbial community structures analysis to determine microbial indicators of contaminants (Obi et al. 2017). Evaluation of the influence of different degrading conditions on microbial communities also revealed significant effects (Techtmann et al. 2017). However, it is worth noting that previous studies were predominantly conducted using simple and stable inlet chemicals, while few investigations have focused on microbial changes with contaminant shifts.

Various VOCs have physical and chemical characteristics that differ significantly. Water-solubility and biodegradability are the two main factors that influence the removal efficiency of biofilters (Deshusses and Webster 2000). Low water-solubility limits mass transfer prior degradation (Alonso et al. 1998), while biodegradability influences the degradation of soluble VOCs. Among all pollutants, hydrocarbons (i.e., alcohols, ketones, esters etc.) have been shown to be the easiest degraded compounds, before alkene and aromatic hydrocarbon (Delhomenie and Heitz 2005). Therefore, different types 
of VOCs alter biofilter performances and microbial community structures significantly. However, precise analyses and conclusive studies are not yet available, especially for waste air treatments.

Few studies have investigated the interactions between environments and microorganisms (Zhang et al. 2018); therefore, the present study was conducted to elucidate these interactions. Specifically, this study investigated a biofilter applied to treat a variety of volatile organic compounds (toluene, ethylbenzene, chlorobenzene, acetone, isopropyl alcohol, ethyl acetate, $\mathrm{n}$-hexane and tetrahydrofuran) in sequence. Differences in degrading performance and the changes in microbial structures during the process were then evaluated. The data obtained in this study will provide insight into microbial community function, functional diversity, and other aspects of the biofilter operation.

\section{Materials and methods}

\section{Biofilter configuration and operation}

\section{Biofilter set up}

The system had a height of $335 \mathrm{~mm}$ and an internal diameter of 118, giving an effective height of packing material of $150 \mathrm{~mm}$ with an approximately $1.7 \mathrm{~L}$ volume (Additional file 1: Figure S1). The air flow was pressurized and controlled with an electromagnetic air compressor (ACO-318, Hailea Co., Ltd., Guangdong, China) and a flowmeter (LZB-WB, Zhenxing Flowmeter Factory, China). Pressurized airflow entered the mixing chamber, which contained multiple vials of evaporating VOCs. The quantity of the vials, volume and volatile area of the VOCs was used to control the inlet concentration. Mixed air flow then entered the biofilter from the bottom and passed through the packing materials containing the microorganisms, after which the treated air was discharged from the top of the biofilter. An electromagnetic flowmeter (Iwaki Co., Ltd, EH-B20VC-220R1) was used to control the liquid flow rate and a microcomputer time controlled switch (Toone Co., Ltd., Shanghai, China) was used to control the spraying rate.

Eight common volatile organic compounds were selected in this study for biofilter performances and microbial community analyses. The selected compounds, including aromatic and non-aromatic hydrocarbons, were common industrial materials or organic solvents (Additional file 1: Table S1). Different VOCs were applied one by one in the order toluene, ethylbenzene, chlorobenzene, acetone, isopropyl alcohol, ethyl acetate, $n$-hexane and tetrahydrofuran. The duration of operation for each compound ranged from 12 to 17 days, depending on the time spent to reach a stable state.

\section{Biofilter operation}

The inlet air flow rate was $2.2 \mathrm{~L} \mathrm{~min}^{-1}$ with an empty bed residence time of $46 \mathrm{~s}$. Spraying was conducted for 1 min every $3 \mathrm{~h}$, giving a total sprayed volume of $90 \mathrm{~mL}$. The nutrient medium was renewed every $3-4$ days, the $\mathrm{pH}$ of the medium was controlled at 6.5-7.2, and temperature ranged from 25 to $32{ }^{\circ} \mathrm{C}$.

\section{Nutrient medium and inoculation}

The nutrient medium used consisted of $10 \mathrm{~g} \mathrm{NaNO}_{3}$, $2.56 \mathrm{~g} \mathrm{Na}_{2} \mathrm{HPO}_{3}$ and $1.66 \mathrm{~g} \mathrm{KH}_{2} \mathrm{PO}_{3}$ per $\mathrm{L}$ water, and the $\mathrm{pH}$ values ranged from 6.5 to 7.2. The medium was inoculated with suspended activated sludge, and $1.7 \mathrm{~L}$ packing materials were filled into the biofilter after being soaked in $1 \mathrm{~L}$ of the activated sludge suspension for an hour.

\section{Analytical methods}

\section{VOCs, $\mathrm{CO}_{2}$ and biomass concentration}

The concentrations of the chemicals were analyzed by gas chromatography (GC-14C, Shimadzu Co. Ltd., Shanghai, China). The temperatures of the inlet, column (HICRON HP-1, $50 \mathrm{~m} \times 0.25 \mathrm{~mm}$ ) and detector were $150{ }^{\circ} \mathrm{C}, 100^{\circ} \mathrm{C}$ and $150{ }^{\circ} \mathrm{C}$, respectively.

The concentration of $\mathrm{CO}_{2}$ was tested using a portable $\mathrm{CO}_{2} \mathrm{~m}$ (Testo 535, Testo China Co. Ltd., China), while decreases in pressure were analyzed with a manometer (Testo 512, Testo China Co. Ltd., China).

Biomass in different periods was tested $2 \mathrm{~h}$ after spraying to minimize the influence of sprayed nutrient medium. The growth of biomass was compared among treatments to evaluate increases in microorganisms.

\section{DNA extraction}

DNA extraction was performed during the stable phase using a Fast DNA ${ }^{\mathrm{TM}}$ SPIN Kit for Soil (MP Biomedicals, Canada), after which the extracted DNA was sequenced using the Illumina MiSeq sequencing platform (Novogene Co., Beijing, China) and the 515F (5'-GTGCCA GCAGCCGCGGTAA-3') and 806R (5'-GGACTACCA GGGTATCTAAT- $3^{\prime}$ ) targeting the V4 region of the $16 \mathrm{~S}$ rRNA gene. Each reverse primer contained a 6-bp errorcorrecting unique barcode and PCR amplification was conducted by subjecting the samples to $98^{\circ} \mathrm{C}$ for $5 \mathrm{~min}$, followed by 40 cycles of $94{ }^{\circ} \mathrm{C}$ for $30 \mathrm{~s}, 55{ }^{\circ} \mathrm{C}$ for $30 \mathrm{~s}$ and $72{ }^{\circ} \mathrm{C}$ for $45 \mathrm{~s}$, and then final extension at $72{ }^{\circ} \mathrm{C}$ for $10 \mathrm{~min}$. Sequences were then analyzed using the Illumina MiSeq platform (Novogene Co., Beijing, China).

\section{DNA sequencing}

Illumina MiSeq original DNA sequence data were processed and analyzed by Qiime (http://qiime.org/) and 
UPARSE (http://drive5.com/uparse/). Paired-end reads from the original DNA fragments were combined using FLASH18. All sequences were aligned with the SILVA bacterial 16S rRNA database. Sequences were clustered into operational taxonomic units (OTUs) at a cutoff of 97\% sequence identity, after which the unweighted UniFrac in principal coordinate analysis $(\mathrm{PCoA})$ was determined by Qiime. Some indices (rarefaction curves, Chao, Simpson, Shannon, coverage) were calculated to reveal Alpha diversity using mothur v.1.32. (http:// www.mothur.org).

The Illumina MiSeq sequencing raw data have been deposited in the NCBI Sequence Read Archive database, and the SRA accession is SRP148831.

\section{Biolog test}

BIOLOG ECO Plates and high-throughput sequencing were used in this study to monitor microbial communities. High-throughput sequencing has helped investigators identify changes in the microbial structure and diversity (Alpana et al. 2017).

BIOLOG Eco-plates were used to investigate SoleCarbon-Source Utilization (SCSU) of different microbial communities. Each plate contained a total of 31 carbon sources in triplicate. The suspension samples required a 5 -min resting after they were obtained from the biofilter. Then, the supernatant was diluted to make the $\mathrm{OD}_{600}$ close to 0.05 . Next, $150 \mu \mathrm{L}$ aliquots of the diluted supernatants were added to the wells of the microplate and incubated at $30{ }^{\circ} \mathrm{C}$ for $3-4$ days.

Data from the Biolog experiments were subsequently analyzed for metabolic function of microbial communities. The ability to degrade each of the 31 carbon source was indicated by average well color developments (AWCD):

$$
\operatorname{AWCD}=\frac{\sum_{i=1}^{96}\left(A_{i}-\frac{A_{1}+A_{33}+A_{65}}{3}\right)}{93} .
$$

In this equation, $A_{i}$ refers to the $\mathrm{OD}_{600}$ value in well number "i", among which No. 1, 33, and 66 were blank values.

Metabolic activities were indicated by changes in the AWCD rate (v) obtained in during the rapid growth rate as follows (Choi and Dobbs 1999):

$$
v=\frac{\mathrm{AWCD}_{1}-\mathrm{AWCD}_{0}}{t},
$$

where $A W C D_{0}$ is the AWCD value at the beginning of the rapid growth period, $A W C D_{1}$ is the AWCD value at the end of the rapid growth period and $t$ is the length of the rapid growth period in hours.

The results were then subjected to principal component analysis (PCA) using SPSS 22.

\section{Results}

Removal and mineralization of VOCs

Shifting of inlet VOCs influenced removal efficiencies were observed (Fig. 1). Specifically, significant declines in removal efficiency were obtained after shifting VOCs; however, it recovered several days later, indicating that the system required time to adapt to the new conditions. Differences in molecular structures are known to impact the biodegradability of different compounds. For example, simpler compounds such as ethanol are more readily degraded by microorganisms than more complex materials (Sempere et al. 2008). In the present study, the removal efficiency varied from $18.2 \%$ (chlorobenzene) to $92.3 \%$ (isopropyl alcohol). The maximum elimination capacities of different volatile organic compounds are listed in Table 1. Alcohols, ketones, esters and ethers appeared to be more easily removed, whose eliminated capacities were higher than $600 \mathrm{mg} \mathrm{m}^{-3} \mathrm{~min}^{-1}$. Moreover, the system showed satisfactory performance for degrading toluene $\left(678.11 \mathrm{mg} \mathrm{m}^{-3} \mathrm{~min}^{-1}\right)$ and tetrahydrofuran (401.53 $\left.\mathrm{mg} \mathrm{m}^{-3} \mathrm{~min}^{-1}\right)$. Conversely, chlorobenzene and $n$-hexane were not easily degraded by microorganisms, as indicated by removal efficiencies lower than 30\%. Overall, considerable differences in degradation performance were seen among different VOCs.

Similar results were observed among VOC mineralization rates (Fig. 1). Surprisingly, the mineralization rate of

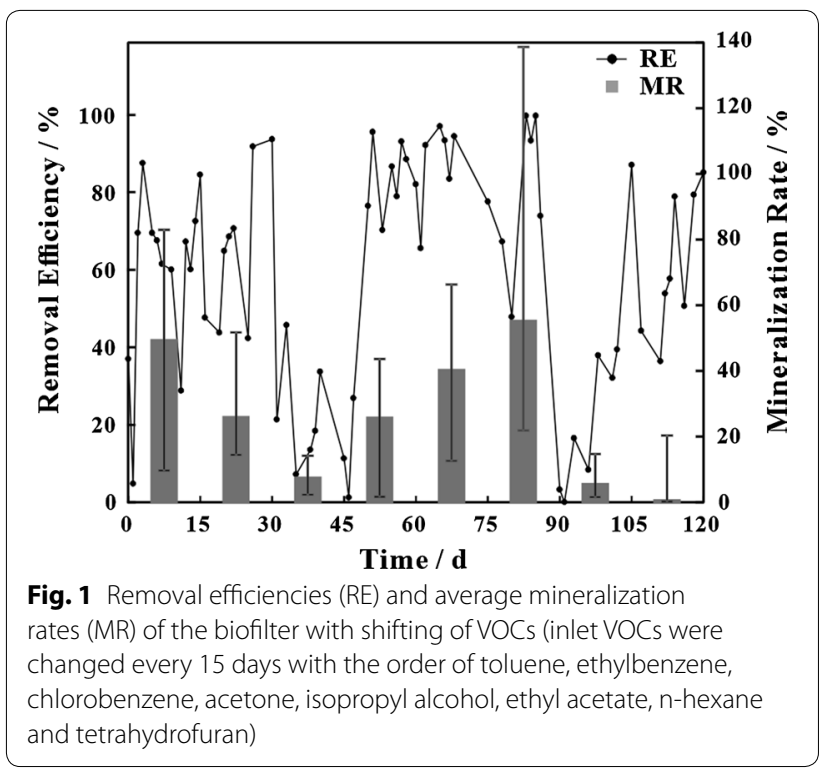


Table 1 Performances of the biofilter with different inlet voCs

\begin{tabular}{lcll}
\hline VOCs & $\begin{array}{l}\text { Inlet } \\
\text { concentration } \\
\left(\mathbf{m g ~ m}^{-\mathbf{3}} \mathbf{)}\right.\end{array}$ & $\begin{array}{l}\text { Removal } \\
\text { efficiency } \\
(\mathbf{\%})\end{array}$ & $\begin{array}{l}\text { Elimination } \\
\text { capacity } \\
\left(\mathbf{m g ~ m}^{-\mathbf{3}} \mathbf{~ m i n}^{\mathbf{- 1}} \mathbf{)}\right.\end{array}$ \\
\hline Toluene & 905 & 57.9 & 678.11 \\
Ethylbenzene & 655 & 67.8 & 574.70 \\
Chlorobenzene & 639 & 18.2 & 150.50 \\
Acetone & 598 & 83.7 & 647.74 \\
Isopropyl alcohol & 839 & 92.3 & 1002.16 \\
Ethyl acetate & 744 & 91.9 & 884.83 \\
n-hexane & 1009 & 29.5 & 385.20 \\
Tetrahydrofuran & 421 & 73.7 & 401.53 \\
\hline
\end{tabular}

tetrahydrofuran was lowest among all tested compounds. More tetrahydrofuran was degraded into intermediates or microorganisms instead of $\mathrm{CO}_{2}$ and $\mathrm{H}_{2} \mathrm{O}$.

Different performance was observed for different inlet VOCs, suggesting that the characteristics of the microbial community changed with inlet VOCs.

\section{Microbial diversity}

The microbial community was analyzed following changes in VOCs (Table 2). The highest OTU value of 690 was observed for toluene, while all other VOCs had OTU values of less than 380 . The ACE and Chao1 index values showed a similar trend, confirming that there was higher microbial diversity when treating toluene.

Shannon's index was lower for compounds of aromatic hydrocarbons, indicating lower microbial diversity. A similar trend was observed for Simpson's index, indicating that a higher microbial diversity led to lower dominance of the dominant species, as occurred with toluene. High consistency was seen between degrading performance and microbial community. As previously mentioned, higher microbial diversity was observed for toluene, which had the highest biodegradability among the aromatic hydrocarbons.
Microbial community structure

During the course of varying inlet VOCs, 21 phyla were found. Ten known phyla and two unidentified phyla were found to account for over $98 \%$ of the abundance (Fig. 2a).

Actinobacteria and Proteobacteria remained dominant throughout the experimental period. Upon further analysis, the abundance of Actinobacteria was found to fluctuate from 17 to $72 \%$, while that of Proteobacteria varied from 19 to $57 \%$. As the inlet VOCs were changed from toluene to ethylbenzene and chlorobenzene, the abundance of Actinobacteria increased in a stepwise fashion to $33.2 \%, 63.9 \%$ and $71.1 \%$, respectively, while that of Proteobacteria decreased from 56.4 to $24.6 \%$ and then $19.8 \%$. Surprisingly, the sum of the two phyla remains stable, with values of $89.6 \%, 88.5 \%$ and $90.9 \%$ being observed for toluene, ethylbenzene and chlorobenzene, respectively.

The changes in the microbial community occurred when the VOCs were switched from aromatic to nonaromatic hydrocarbons. The sum of Actinobacteria and Proteobacteria decreased from $90 \%$ to approximately 60\%-70\%, while the abundances of Planctomycetes and Thermi increased significantly after the hydrocarbons were changed. The abundance of the phylum Planctomycetes increased when some aromatic chemicals were removed. Moreover, their abundance increased when the aromatic hydrocarbons were replaced with non-aromatic chemicals such as acetone. Thermi remained stable during purification of non-aromatic hydrocarbons, fluctuating at levels of $5.0-8.0 \%$. The abundance of Bacteroidetes peaked at $14.6 \%$ in response to acetone and then decreased to $1.0 \%$ upon application of $n$-hexane. These findings indicate that acetone was a better carbon and energy source for species in the phylum Bacteroidetes than n-hexane. The abundance of Cyanobacteria tripled in the presence of tetrahydrofuran when compared to other compounds. It has been speculated that members of the phylum Cyanobacteria could include more tetrahydrofuran degraders than other phyla.

Table 2 Indexes of microbial following treatment with different types of VOCs

\begin{tabular}{|c|c|c|c|c|c|c|}
\hline VOCs & OTU & ACE & Chao1 & Coverage & Shannon & Simpson \\
\hline Toluene & 690 & 998.41 & 971.64 & 0.9959 & 4.73 & 0.9062 \\
\hline Ethylbenzene & 357 & 487.79 & 459.24 & 0.9976 & 3.34 & 0.7778 \\
\hline Chlorobenzene & 372 & 499.56 & 473.51 & 0.9969 & 3.28 & 0.7549 \\
\hline Acetone & 357 & 414.83 & 395.65 & 0.9963 & 5.03 & 0.9322 \\
\hline Isopropyl alcohol & 335 & 423.24 & 404.93 & 0.9976 & 4.39 & 0.8965 \\
\hline Ethyl acetate & 357 & 404.22 & 392.05 & 0.9974 & 5.13 & 0.9248 \\
\hline N-hexane & 285 & 364.87 & 359.59 & 0.9977 & 5.14 & 0.9481 \\
\hline Tetrahydrofuran & 312 & 374.90 & 356.29 & 0.9976 & 5.06 & 0.9446 \\
\hline
\end{tabular}




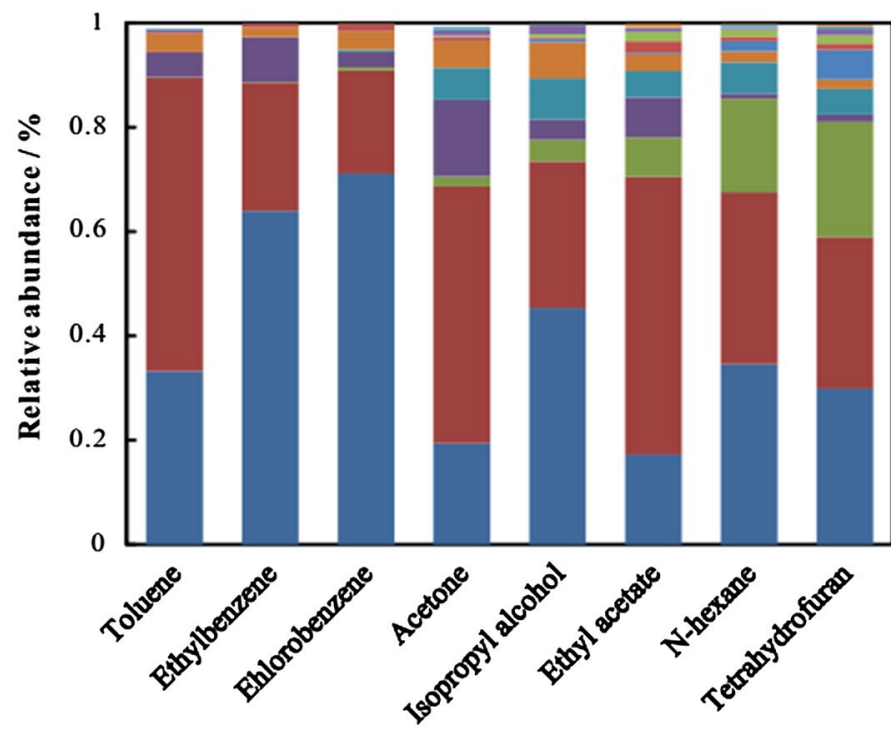

a

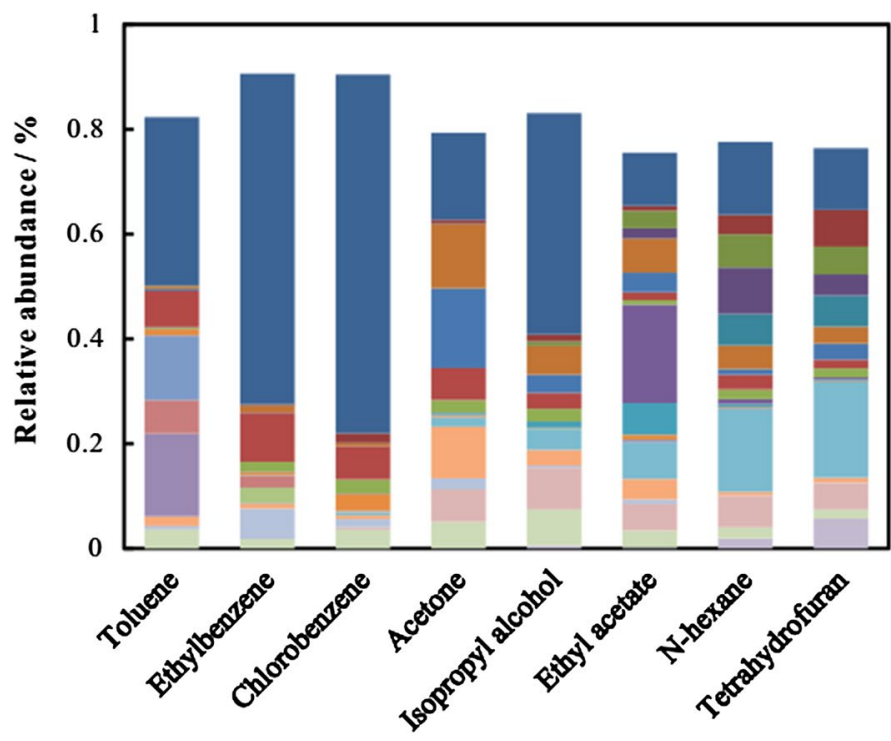

b
Acidobacteria

Chloroflexi

nunassigned;Other

घemmatimonadetes

- Firmicutes

- Cyanobacteria

None; Other

a Thermi

Bacteroidetes

aPlanctomycetes

-Proteobacteria

actinobacteria
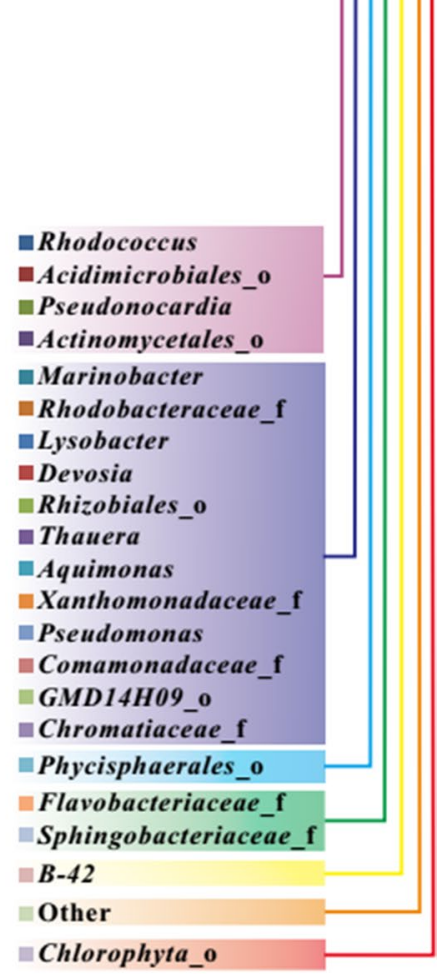

Fig. 2 Figure 3-2 Microbial community structures (a relative abundances of microorganisms at the phylum level following the inlet of different volatile organic compounds; $\mathbf{b}$ relative abundances at the genus level when treating different volatile organic compounds. 22 genera were included, including the five genera with the highest abundance in each stage)

A total of 264 genera were obtained during the operation, 21 of which were present in high abundance. The sum of these dominant genera accounted for $75 \%-91 \%$ of the total biomass (Fig. 2b). All genera belonged to the top seven phyla, with most belonging to the phyla Actinobacteria and Proteobacteria. Four genera, including Rhodococcus, Pseudonocardia and other two unidentified genera from the phylum Actinobacteria, had high abundances. Among them, Rhodococcus had significant advantages in abundance throughout the experimental period. The phylum Proteobacteria, which contained Pseudomonas, Devosia, Aquimonas, Marinobacter and eight other genera, showed high stability and uniformity with increased abundance when treating various contaminants, as opposed to genera belonging to the phylum Actinobactria.

The genus Rhodococcus, which belongs to the phylum Actinobacteria, was obtained in high abundance (>10\%) throughout the operation. Surprisingly, higher values of relative abundance were observed when aromatic 
hydrocarbons were being eliminated. Similarly, the abundance of Devosia sp. increased in response to the inlet of aromatic hydrocarbons. During the operation, specific degraders were identified. Specifically, high abundance (18.8\%) of Thauera was observed in the presence of ethyl acetate, while less than $1 \%$ was observed in the presence of other VOCs. In addition, the genus Pseudomonas showed highest abundance during the inlet of toluene, while it comprised less than $0.5 \%$ in the presence of other VOCs.

\section{Microbial metabolic profile}

The values of average well color development (hereafter AWCD, Fig. 3) were affected by density and activity of bacterial community (Preston-Mafham et al. 2002; Rutgers et al. 2016). The results showed that carbon source metabolic capacity of the microbial community decreased gradually with time. Initially, the AWCD values of toluene and ethylbenzene were higher than those observed for other VOCs. The rate of AWCD increase was $0.042 \mathrm{~cm}^{-1} \mathrm{~h}^{-1}$ for toluene and $0.035 \mathrm{~cm}^{-1} \mathrm{~h}^{-1}$ for ethylbenzene, which illustrated that the microbial community had higher activity in the presence of purified toluene than ethylbenzene. Growth rate declined to approximately $0.025 \mathrm{~cm}^{-1} \mathrm{~h}^{-1}$ when treating acetone and chlorobenzene and carbon source metabolic capacities and activities declined with time. The increase rate of AWCD when treating isopropyl alcohol, ethyl acetate, hexane and tetrahydrofuran were $0.013 \mathrm{~cm}^{-1} \mathrm{~h}^{-1}$, $0.014 \mathrm{~cm}^{-1} \mathrm{~h}^{-1}, 0.010 \mathrm{~cm}^{-1} \mathrm{~h}^{-1}$ and $0.015 \mathrm{~cm}^{-1} \mathrm{~h}^{-1}$, respectively. Taken together, these results suggested that some measures might be taken to increase the activities of microorganisms after a long-term operation.

The duration between hours 20 and 30 was a period when the highest AWCD increasing rates were obtained. Therefore, principle component analysis was conducted

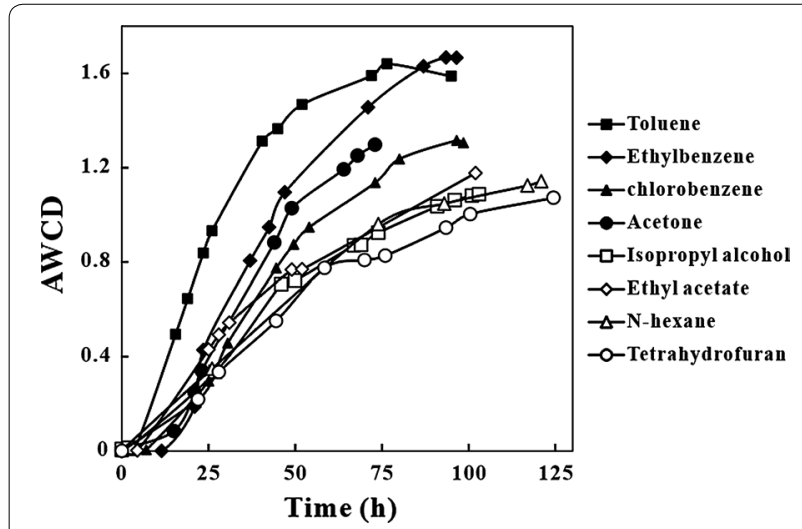

Fig. 3 AWCD changes in microbial community under different inlet VOCS in this study to obtain further insight (Fig. 4) based on data obtained between hours 20 and 30 .

\section{Discussion}

The dominant phyla Actinobacteria and Proteobacteria are the most commonly reported prokaryotic degraders (Coleman et al. 2006). As reported in previous studies, many members of the phylum Actinobacteria have aromatic hydrocarbon degrading abilities, and some species can utilize complicated chemicals as carbon or energy sources for growth; however, the capability for degradation decreases with increasing carbon chain length (Wen et al. 2014; Zylstra et al. 2000). Proteobacteria has previously been widely applied in bioremediation for hydrocarbon purification in combination with Actinobacteria, which is another dominant phylum commonly seen in aromatic hydrocarbon biodegradation (Fuentes et al. 2014). Hence, we can conclude that Actinobacteria and Proteobacteria played crucial roles in aromatic hydrocarbon purification in this study. Increasing abundance of the phylum Planctomycetes when some aromatic chemicals were being removed have also been confirmed in previous study (Delgado-Balbuena et al. 2016).

On genus level, it was previously reported that Rhodococcus can utilize and removal a large variety of pollutants (Maia et al. 2018; Warhurst and Fewson 1994). Members of the genus Rhodococcus were found to have the considerable ability to degrade a great number of aromatic hydrocarbons several decades ago (Sorkhoh et al. 1990). As well-known VOCs degraders, Rhodococcus species can utilize various chemicals and have therefore been applied frequently in VOCs purifications ( $\mathrm{Li}$ et al. 2016). It was also confirmed that Rhodococcus species can degrade complicated chemicals like three to five

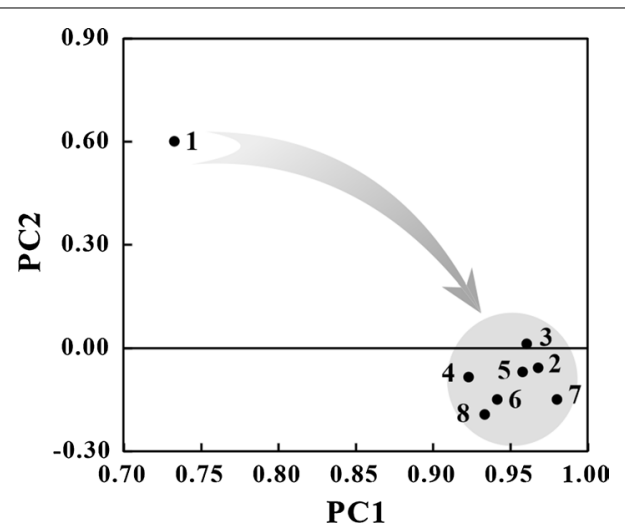

Fig. 4 Carbon metabolism principle component analysis of microbial community with different inlet VOCs: No. 1-8 referred to stages of toluene, ethylbenzene, chlorobenzene, acetone, isopropyl alcohol, ethyl acetate, $n$-hexane and tetrahydrofuran 
rings polycyclic aromatic hydrocarbons (PAHs) (Song et al. 2011). Moreover, some strains of Rhodococcus were shown to have broad degradation capacities toward a mixture of 16 VOCs including benzene, toluene, ethylbenzene, $m$-xylene, $p$-xylene, $o$-xylene, and octane (Auffret et al. 2009). Because of its efficient and broad degradation capacities, Rhodococcus was used to remediate heavily PAH-contaminated soil with total PAHs of $375 \mathrm{mg}$, and up to $55 \%$ was removed (Sun et al. 2012). These performances could explain the high abundance of Rhodococcus throughout the experimental period and likely contributed greatly to the biodegradation performance in this system.

In consideration of other genera, the findings in this study are consistent with those of previous reports. High abundances of Devosia sp. were previously observed in aromatic compounds purification systems (Ramos et al. 2015), and they have been used as aromatic compounds degraders in previous studies (Papale et al. 2017). Ethyl acetate can be utilized as carbon resources by Thauera sp. (Du et al. 2017). The genus Pseudomonas was previously reported to be a toluene utilizer and degrader (Hernandez and Torre 2011; Su et al. 2014). Pseudomonas sp. was previously proposed to be indicators of biodegradation because of their sensitivity to substrate change (Obi et al. 2017; Yakimov et al. 2007).

The significant differences observed in this study indicated that target VOCs had significant effects on microorganisms, particularly at the genus level. The population of utilizers and degraders increased rapidly in the response to VOCs, with some becoming dominant species. Subsequently, changes in inlet VOCs caused dramatic decreases shifts in these populations. Taken together, these results indicate that the microbial community has the ability to adapt to new environmental conditions. Accordingly, there is the potential to develop specific measures to facilitate such adaptation to optimize biodegradation, such as increasing the abundance of specific degraders artificially.

The results revealed that the microbial community was significantly different when purifying toluene then when treating other VOCs. Surprisingly, the communities were highly similar in the presence of all other test compounds. This might have occurred because of significant microbial community shifts in the initial operations (Qiu et al. 2013). After the initial operation, the system became relatively stable, possibly indicating that the community stabilized with time.

Biodegradability of different VOCs has remarkable influences on microbial elimination performance, community structures and metabolic profiles. The highest diversity was obtained at the beginning of the operation while eliminating toluene. Diversity declined with prolonged operation, and significant differences were found in microbial community structures at both the phylum and genus levels. As the experiment continued, the carbon source metabolic capacity declined gradually. The metabolic characteristics of carbon source utilization differed significantly following toluene input, while it was similar among all other treatment groups. Artificial addition of degraders and measures to increase microbial activity might optimize biofiltration.

\section{Additional file}

Additional file 1: Figure S1. Biofilter structure and the flow directions of air/water flow. Table S1. The characteristics of the selected VOCs.

\section{Authors' contributions}

JX conceived the original idea and supervise the project. GW, MY and LL completed the experiment and data analysis. LL wrote the draft of the manuscript. All authors discussed the results and contribute to the final manuscript. All authors read and approved the final manuscript.

\section{Author details}

${ }^{1}$ Environmental Simulation and Pollution Control State Key Joint Laboratory, School of Environment, Tsinghua University, Beijing, China. ${ }^{2}$ Graduate School at Shenzhen, Tsinghua University, Shenzhen, China. ${ }^{3}$ Central Research Institute of Building and Construction, MCC Group, Co, Ltd., Beijing, China.

\section{Acknowledgements}

This study was supported by the National Natural Science Foundation of China (No. 51378286). This study was also supported by the Science and Technology Program for Water Pollution Control (No. 2011ZX07301-003). Help from the State Environmental Protection Key Laboratory of Microorganism Application at Tsinghua University is appreciated. Thanks are given to Dr. Guo Qian for her linguistic assistance during the preparation of this manuscript, and the anonymous reviewers for their helpful comments regarding the manuscript. We would like to thank LetPub (http://www.letpub.com) for providing linguistic assistance during the preparation of this manuscript.

\section{Competing interests}

The authors declare that they have no competing interests.

\section{Availability of data and materials}

All datasets supporting the conclusion of the manuscript were included in the manuscript.

\section{Consent for publication}

All authors gave their consent for publication.

\section{Ethics approval and consent to participate}

This article does not contain any studies with human participants or animals performed by any of the authors.

\section{Funding}

This work has been supported by the National Natural Science Foundation of China (No. 51378286) and the Science and Technology Program for Water Pollution Control (No. 2011ZX07301-003)

\section{Publisher's Note}

Springer Nature remains neutral with regard to jurisdictional claims in published maps and institutional affiliations. 
Received: 15 September 2018 Accepted: 25 September 2018

Published online: 03 October 2018

\section{References}

Alonso C, Suidan MT, Kim BR, Kim BJ (1998) Dynamic mathematical model for the biodegradation of VOCs in a biofilter: biomass accumulation study. Environ Sci Technol 32(20):3118-3123

Alpana S, Vishwakarma P, Adhya TK, Inubushi K, Dubey SK (2017) Molecular ecological perspective of methanogenic archaeal community in rice agroecosystem. Sci Total Environ 596:136-146

Auffret M, Labbe D, Thouand G, Greer CW, Fayolle-Guichard F (2009) Degradation of a mixture of hydrocarbons, gasoline, and diesel oil additives by Rhodococcus aetherivorans and Rhodococcus wratislaviensis. Appl Environ Microbiol 75(24):7774-7782

Cheng ZW, Lu LC, Kennes C, Ye JX, Yu JM, Chen DZ, Chen JM (2016) A composite microbial agent containing bacterial and fungal species: optimization of the preparation process, analysis of characteristics, and use in the purification for volatile organic compounds. Bioresour Technol 218:751-760

Choi KH, Dobbs FC (1999) Comparison of two kinds of Biolog microplates (GN and E(O) in their ability to distinguish among aquatic microbial communities. J Microbiol Methods 36(3):203-213

Coleman NV, Bui NB, Holmes AJ (2006) Soluble di-iron monooxygenase gene diversity in soils, sediments and ethene enrichments. Environ Microbiol 8(7):1228-1239

Delgado-Balbuena L, Bello-Lopez JM, Navarro-Noya YE, Rodriguez-Valentin A Luna-Guido ML, Dendooven L (2016) Changes in the bacterial community structure of remediated anthracene-contaminated soils. PLoS ONE 11(10):e0160991

Delhomenie MC, Heitz M (2005) Biofiltration of air: a review. Crit Rev Biotechnol 25(1-2):53-72

Deshusses MA Webster TS (2000) Construction and economics of a pilot/fullscale biological trickling filter reactor for the removal of volatile organic compounds from polluted air. J Air Waste Manage 50(11):1947-1956

Du R, Cao SB, Li BK, Niu M, Wang SY, Peng YZ (2017) Performance and microbial community analysis of a novel DEAMOX based on partialdenitrification and anammox treating ammonia and nitrate wastewaters. Water Res 108:46-56

Fuentes S, Mendez V, Aguila P, Seeger M (2014) Bioremediation of petroleum hydrocarbons: catabolic genes, microbial communities, and applications. Appl Microbiol Biotechnol 98(11):4781-4794

Hernandez M, Torre RM (2011) Long-term influence of the presence of a non-aqueous phase on the cell surface hydrophobicity of Pseudomonas in two-phase partitioning bioreactors. Appl Microbiol Biotechnol 89(5):1573-1581

Khan Fl, Ghoshal AK (2000) Removal of volatile organic compounds from polluted air. J Loss Prev Process Ind 13(6):527-545

Li C, Zhang CY, Song GL, Liu H, Sheng GH, Ding ZF, Wang ZL, Sun Y, Xu Y, Chen $J$ (2016) Characterization of a protocatechuate catabolic gene cluster in Rhodococcus ruber OA1 involved in naphthalene degradation. Ann Microbiol 66(1):469-478

LiY, Hua Q, Chen CH, Wang XL, Gao DW (2017) Performance and microbial community structure in an integrated anaerobic fluidized-bed membrane bioreactor treating synthetic benzothiazole contaminated wastewater. Bioresour Technol 236:1-10

Maia AS, Tiritan ME, Castro PML (2018) Enantioselective degradation of ofloxacin and levofloxacin by the bacterial strains Labrys portucalensis F11 and Rhodococcus sp FP1. Ecotox Environ Safe 155:144-151

Obi CC, Adebusoye SA, Amund OO, Ugoji EO, Ilori MO, Hedman CJ, Hickey WJ (2017) Structural dynamics of microbial communities in polycyclic aromatic hydrocarbon-contaminated tropical estuarine sediments undergoing simulated aerobic biotreatment. Appl Microbiol Biotechnol 101(10):4299-4314
Papale M, Giannarelli S, Francesconi S, Di Marco G, Mikkonen A, Conte A, Rizzo C, De Domenico E, Michaud L, Lo Giudice A (2017) Enrichment, isolation and biodegradation potential of psychrotolerant polychlorinated-biphenyl degrading bacteria from the Kongsfjorden (Svalbard Islands, High Arctic Norway). Mar Pollut Bull 114(2):849-859

Preston-Mafham J, Boddy L, Randerson PF (2002) Analysis of microbial community functional diversity using sole-carbon-source utilisation profiles-a critique. FEMS Microbiol Ecol 42(1):1-14

Qiu GL, Song YH, Zeng P, Duan L, Xiao SH (2013) Combination of upflow anaerobic sludge blanket (UASB) and membrane bioreactor (MBR) for berberine reduction from wastewater and the effects of berberine on bacterial community dynamics. J Hazard Mater 246:34-43

Ramos C, Suarez-Ojeda ME, Carrera J (2015) Long-term impact of salinity on the performance and microbial population of an aerobic granular reactor treating a high-strength aromatic wastewater. Bioresour Technol 198:844-851

Rutgers M, Wouterse M, Drost SM, Breure AM, Mulder C, Stone D, Creamer RE, Winding A, Bloem J (2016) Monitoring soil bacteria with community-level physiological profiles using Biolog (TM) ECO-plates in the Netherlands and Europe. Appl Soil Ecol 97:23-35

Sempere F, Gabaldon C, Martinez-Soria V, Marzal P, Penya-Roja JM, AlvarezHornos FJ (2008) Performance evaluation of a biotrickling filter treating a mixture of oxygenated VOCs during intermittent loading. Chemosphere 73(9):1533-1539

Song XH, Xu Y, Li GM, Zhang Y, Huang TW, Hu Z (2011) Isolation, characterization of Rhodococcus sp P14 capable of degrading high-molecular-weight polycyclic aromatic hydrocarbons and aliphatic hydrocarbons. Mar Pollut Bull 62(10):2122-2128

Sorkhoh NA, Ghannoum MA, Ibrahim AS, Stretton RJ, Radwan SS (1990) CrudeOil and hydrocarbon-degrading strains of rhodococcus-rhodochrous isolated from soil and marine environments in Kuwait. Environ Pollut 65(1):1-17

Su Y, Xia FF, Tian BH, Li W, He R (2014) Microbial community and function of enrichment cultures with methane and toluene. Appl Microbiol Biotechnol 98(7):3121-3131

Sun GD, Xu Y, Jin JH, Zhong ZP, Liu Y, Luo M, Liu ZP (2012) Pilot scale ex situ bioremediation of heavily PAHs-contaminated soil by indigenous microorganisms and bioaugmentation by a PAHs-degrading and bioemulsifierproducing strain. J Hazard Mater 233:72-78

Techtmann SM, Zhuang MB, Campo P, Holder E, Elk M, Hazen TC, Conmy R, Domingo JWS (2017) Corexit 9500 enhances oil biodegradation and changes active bacterial community structure of oil-enriched microcosms. Appl Environ Microbiol. https://doi.org/10.1128/AEM.03462-16

Warhurst AM, Fewson CA (1994) biotransformations catalyzed by the genus Rhodococcus. Crit Rev Biotechnol 14(1):29-73

Wen ZD, Gao DW, Wu WM (2014) Biodegradation and kinetic analysis of phthalates by an Arthrobacter strain isolated from constructed wetland soil. Appl Microbiol Biotechnol 98(10):4683-4690

Yakimov MM, Timmis KN, Golyshin PN (2007) Obligate oil-degrading marine bacteria. Curr Opin Biotechnol 18(3):257-266

Zhang Y, Sun R, Zhou AJ, Zhang JG, Luan YB, Jia JN, Yue XP, Zhang J (2018) Microbial community response reveals underlying mechanism of industrial-scale manganese sand biofilters used for the simultaneous removal of iron, manganese and ammonia from groundwater. Amb Express. https //doi.org/10.1186/s13568-017-0534-7

Zheng JY, Yu YF, Mo ZW, Zhang Z, Wang XM, Yin SS, Peng K, Yang Y, Feng XQ, Cai HH (2013) Industrial sector-based volatile organic compound (VOC) source profiles measured in manufacturing facilities in the Pearl River Delta, China. Sci. Total Environ. 456:127-136

Zylstra GJ, Goyal AK, Cigolini JF, Dennis JD (2000) Bioprospecting for novel aromatic oxygenases. Abstracts of Papers of the American Chemical Society 219:U158-U158 